\title{
La tabla periódica y sus patrones para la predicción del comportamiento fisicoquímico
}

\author{
Luis Hernández Adame, Pablo Luis Hernández-Adame, \\ Jaime Ruiz-García y Héctor René Vega-Carrillo
}

\begin{abstract}
Resumen
Conocer las características fisicoquímicas de los elementos es muy importante, ya que éstas nos permiten estudiar sus propiedades, analizar su comportamiento y hasta sintetizar nuevos elementos. En este sentido, este trabajo pretende explorar, de un modo sencillo y didáctico, la estructura de la tabla periódica, la manera de interpretarla y cómo encontrar los patrones de predicción que describen el comportamiento fisicoquímico de los elementos. Este tema es de interés para los lectores con curiosidad por las ciencias y los ayudará a comprender el significado de toda esta valiosa información.
\end{abstract}

Palabras clave: elementos químicos, tabla periódica, predicción fisicoquímica.

\section{The Periodic Table and its Patterns to Predict Physicochemical Behavior}

\begin{abstract}
It is important to know the physicochemical characteristics of the elements, since they allow us to study their properties, analyze their behavior, and even synthesize new elements. In this sense, this work describes the periodic table, in a simple and didactic way, a method for its easy interpretation, and how we can find the prediction patterns that describe the physicochemical behavior of the elements. This topic is for curious readers who intend to study Science and it will help them understand the meaning of all this valuable information.
\end{abstract}

Keywords: Chemical Elements, Periodic Table, Physicochemical Prediction.

Recepción: 15/11/2019. Aprobación: 28/07/2020. Dol: http://doi.org/10.22201/cuaieed.16076079e.2020.21.6.5 
Luis Hernández Adame

ladame@cibnor.mx orcid.org/0000-0002-0188-7526 https://www.cibnor.gob.mx/cvpersonal?JOS\&CIB_ID=09017

Es investigador en el CIBNOR. Doctor en Ciencia de Materiales por la FCQ-UASLP y miembro del sNI nivel 1. Sus líneas de investigación se enfocan al desarrollo de nanomateriales y nanotecnología, aplicados en la salud animal, vegetal y humana.

Sus proyectos vigentes son el desarrollo de biosensores, vehículos de transporte para biomoléculas (nanovacunas) y nanoestructuras usadas en sistemas agrícolas.

\section{Pablo Luis Hernández-Adame}

pabloyae_2@hotmail.com orcid.org/0000-0003-3554-5898

Es estudiante de doctorado en el IF de la UASLP. Su trabajo doctoral se enfoca en el estudio del colesterol en la distribución del receptor de neurocininas Nk-1 en la membrana plasmática de células HEk-293 por medio de técnicas espectroscópicas. Además, realiza investigación de la dinámica y estabilidad de balsas lipídicas en membranas modelo, por medio de la técnica de monocapas de Langmuir y Microscopio de Ángulo de Brewster (BAM).

\section{Jaime Ruiz-García}

jaime@mail.ifisica.uaslp.mx orcid.org/0000-0003-3730-3825

Es Profesor-Investigador del if en la UASLP. Doctor en Física por The University of Maryland. Miembro del sNi nivel 3. Sus líneas de investigación se enfocan en el área de biofísica: sus proyectos actuales son el estudio de moléculas de interés biológico (membranas modelo, DNA, RNA, autoensamblamiento de proteínas, virus) para su uso en medicina.

\section{Héctor René Vega-Carrillo}

fermineutron@yahoo.com orcid.org/0000-0002-7081-9084

Es profesor-investigador en la Uaz. Es Doctor of Philosophy (Nuclear Engineering) por The University of Texas at Austin. Es miembro del sNI nivel 2. Sus líneas de investigación son la espectrometría y dosimetría de neutrones. 


\section{La tabla periódica y los elementos}

La tabla periódica es una poderosa herramienta que representa de forma gráfica todos los símbolos de los elementos químicos que componen la materia que conocemos. Fue estructurada por dos científicos que trabajaban simultáneamente; uno de ellos fue el químico y médico alemán Julius Lothar Meyer, que en 1968 construyó una tabla que relacionaba el volumen y masa atómica de los elementos con su comportamiento químico. Sin embargo, su descubrimiento fue poco reconocido, ya que el químico ruso Dmitri Mendeléyev presentó una teoría muy similar en 1869 y se llevó casi todos los honores (Ramos, 2017). Mendeléyev propuso que todos los elementos conocidos hasta entonces seguían un patrón de comportamiento fisicoquímico conforme aumentaba la masa atómica del elemento; posteriormente, a este patrón se le dio el nombre de ley periódica. Años más tarde, en 1913, el físico Henry Moseley (estudiante del reconocido investigador Ernest Rutherford) realizó una serie de experimentos irradiando los elementos metálicos con rayos $X$, y descubrió que su estabilidad y comportamiento químico eran mejor representados siguiendo su número atómico, razón por la cual, decidió reordenar la tabla y estructurarla a la manera que actualmente la conocemos.

Como primer acercamiento, podemos decir que la tabla periódica está formada por una agrupación de cuadros que están ordenados en columnas conocidas como familias o grupos, y renglones llamados períodos, como se representa en la figura 1.

Figura 1. Representación esquemática de la tabla periódica, que muestra cada elemento, el bloque al que pertenecen, y su división en grupos y períodos.

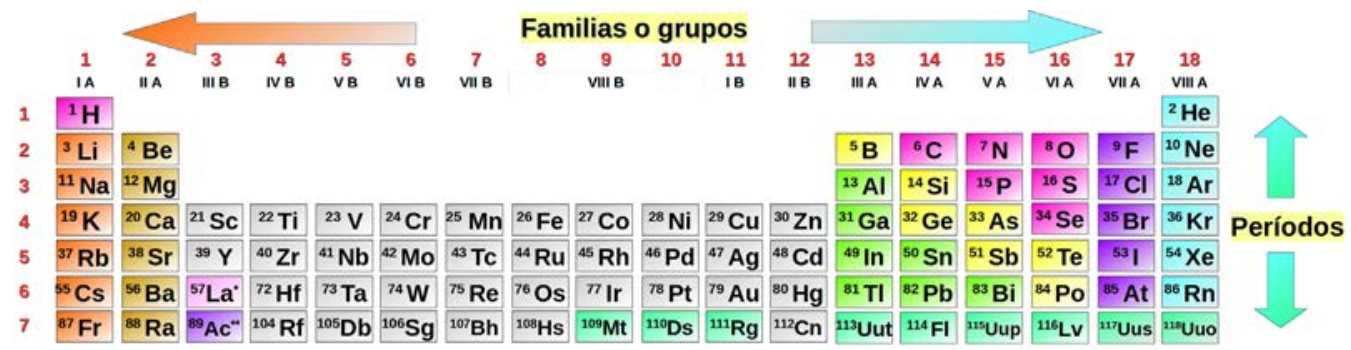

* ${ }^{50} \mathrm{Ce}{ }^{59} \mathrm{Pr}{ }^{60} \mathrm{Nd}{ }^{61} \mathrm{Pm}{ }^{62} \mathrm{Sm}{ }^{63} \mathrm{Eu}{ }^{64} \mathrm{Gd}{ }^{65} \mathrm{~Tb}{ }^{66} \mathrm{Dy}{ }^{67} \mathrm{Ho}{ }^{68} \mathrm{Er}{ }^{69} \mathrm{Tm}{ }^{70} \mathrm{Yb}{ }^{71} \mathrm{Lu}$

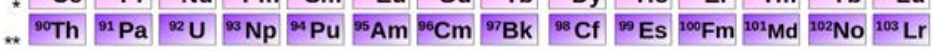

\begin{tabular}{c|c|c|c|c|c|c|}
\hline $\begin{array}{c}\text { No } \\
\text { metales }\end{array}$ & $\begin{array}{c}\text { Metales } \\
\text { alcalinos }\end{array}$ & Alcalinotérreos Lantánidos & Actínidos & $\begin{array}{l}\text { Metales de } \\
\text { transición }\end{array}$ & Transuránicos Metales & Metaloides Halógenos $\begin{array}{c}\text { Gases } \\
\text { nobles }\end{array}$ \\
\hline
\end{tabular}

Los cuadros de la tabla periódica almacenan la información perteneciente a cada uno de los elementos. Actualmente la componen 118 (Karol et al., 2016), los cuales, según el formato de la IUPAC (International Union of Pure and Applied Chemistry), están representados en el interior de cada cuadro por un símbolo químico de una o más letras que, en la mayoría de los casos, deriva de su nombre en inglés, por ejemplo, el oxígeno representado por la letra $O$ (oxygen). O bien, se usan algunos otros símbolos que provienen de la raíz en otras lenguas, como el oro y la plata, representados por las letras $A u$ y $A g$, debido a que derivan de las palabras en latín aurum y argentum, respectivamente. Asimismo, en la parte superior del símbolo, 
Figura 2. Símbolo que representa los elementos químicos y sus principales datos en la tabla periódica. encontramos el número atómico, que nos indica el número de protones que contiene el núcleo de cada elemento; mientras que, en la parte inferior, está el nombre del elemento y su masa atómica, la masa promedio que tiene cada elemento en el núcleo (protones + neutrones), y que además considera sus isótoposy su abundancia porcentual en la naturaleza, como se indica en la figura 2.

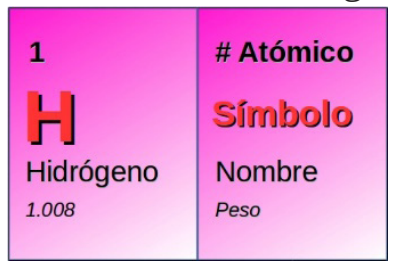

\section{Distribución de los elementos dentro de la tabla}

La tabla periódica está dividida en diferentes bloques o series de elementos que comprenden uno o más grupos. Éstos contienen elementos con un comportamiento químico similar y están clasificados en metales, metaloides y no metales. Dentro del grupo de los metales, se encuentran los metales alcalinos, alcalinotérreos, lantánidos, actínidos y los metales de transición, que se caracterizan de manera general, y al contrario de los no metales, por tener un aspecto brillante, ser buenos conductores del calor y la electricidad, además de que según su número atómico podemos predecir si tienen baja o alta densidad, su grado de dureza y si son altamente reactivos. Por otra parte, en el grupo de los metaloides (también conocidos como semimetales) podemos encontrar los elementos que tienen un comportamiento intermedio entre los metales y los no metales, además de aquellos con muy baja reactividad química que comprenden los gases nobles pertenecientes al grupo 18 o también conocido como grupo vilA según su antigua clasificación. Por último, mencionaremos a los transuránicos: los últimos elementos que se han descubierto (del 93 al 118); tienen un gran número atómico y casi todos han sido generados de manera artificial en investigaciones científicas; su comportamiento químico es extremadamente raro.

\section{Interpretación de la tabla y patrones de comportamiento fisicoquímico}

Una característica muy importante de la tabla periódica es que en ella es posible encontrar patrones que nos predicen con precisión algunas propiedades fisicoquímicas de cada elemento. Para esto, bastará con ubicar la posición y determinar el grupo y perío do al que pertenecen. Algunas de las propiedades que son fácilmente reconocibles son: la configuración electrónica de valencia, el radio atómico, el potencial de ionización, la afinidad electrónica y la electronegatividad, entre algunas otras. Estas propiedades son muy importantes, ya que nos permiten relacionar a los elementos de acuerdo con su comportamiento, para generar nuevos elementos y materiales. 
Figura 3. Representación del orden creciente del número atómico y la densidad electrónica. Cada punto alrededor del símbolo químico representa el número de electrones del último orbital, conocidos como electrones de valencia

El estado de oxidación es un número que indica de forma indirecta, el número de electrones y la carga que tiene un átomo. Este valor es muy útil para los procesos de oxidación y reducción.
Para su interpretación, partiremos por leer la tabla periódica en orden creciente del número atómico. Para esto, recordemos que, en un átomo estable, conforme se incrementa el número de protones, lo hace también el número electrones en la misma cantidad. Así, al leer la tabla periódica de izquierda a derecha, y de arriba hacia abajo, notaremos un aumento en la densidad electrónica, además de que, todos los elementos pertenecientes al grupo 1 (IA) únicamente tendrán un electrón en su último orbital; los ubicados en el grupo 2 (॥A), dos electrones; y así sucesivamente con excepción de los grupos 3 (IIB) al 12 (॥B), como se muestra en la figura 4, ya que éstos corresponden a los metales de transición y pueden tener múltiples estados de oxidación estables ${ }^{1}$ (valencia), debido a la facilidad con la que pierden electrones de su orbital d (Scerri, 2007).

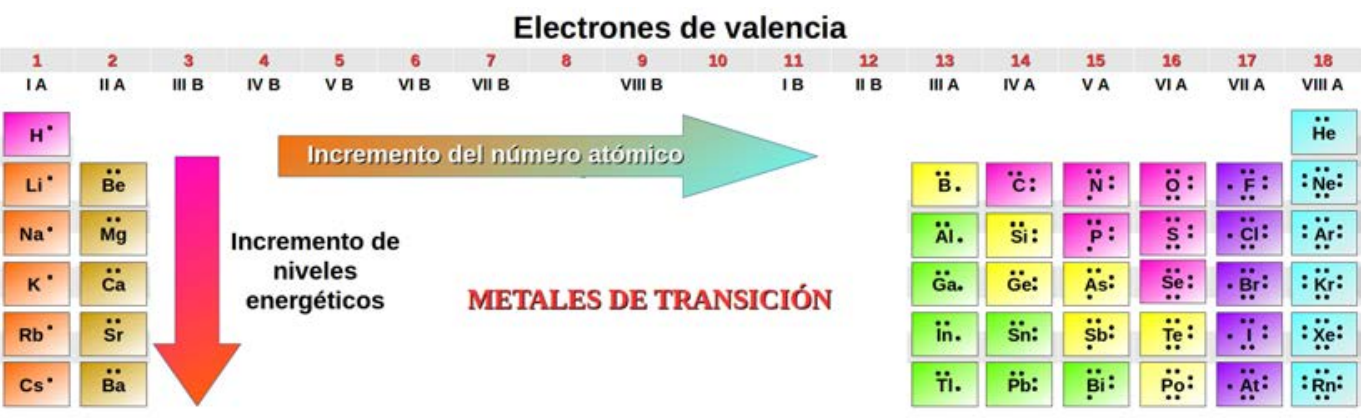

Esta característica en la configuración electrónica de valencia nos permite predecir la capacidad de ionización y la probabilidad de estos elementos para donar o recibir electrones durante las reacciones químicas; esto es, entre menos electrones tenga el orbital (elementos de la parte izquierda de la tabla), más fácil será compartirlos. En este sentido, podemos decir que si nos movemos en la tabla periódica de izquierda a derecha, aumentamos de forma gradual el número de electrones en el último orbital; así, por ejemplo, los elementos del grupo 17 (vIIA), los que tienen un orbital de valencia más Ileno (7 electrones), presentan una mayor tendencia a recibir electrones. Por otra parte, los elementos pertenecientes a los gases nobles son otro caso excepcional, ya que mantienen tendencia a no participar en reacciones químicas debido a que su octeto de electrones del último orbital se considera completo.

El período es otro factor importante que debemos entender en la tabla. Cada renglón representa un nivel energético ocupado por los electrones, es decir, los elementos ubicados en el período 1 tendrán distribuidos sus electrones en un solo nivel energético, los ubicados en el período 2 tendrán dos niveles energéticos y así sucesivamente, por lo que los elementos del período 7 son los de mayor densidad electrónica, distribuida en 7 orbitales.

Al tener en cuenta la información provista por los grupos y períodos, es posible predecir el incremento del radio atómico de los elementos, para el cual es necesario considerar dos factores: 1) el número de electrones y sus niveles energéticos, y 2) las fuerzas electromagnéticas de atracción que ejerce el núcleo 
Figura 4. Representación esquemática de los patrones de predicción del comportamiento fisicoquímico de los elementos en la tabla periódica. Cada flecha representa el incremento del radio atómico, la energía de ionización, la afinidad electrónica y la electronegatividad de los elementos, de acuerdo con su posición en la tabla. sobre los electrones. Un esquema representativo se ilustra en la figura 3. Para explicar esto, debemos tener en mente que al aumentar el número de electrones, también lo hacen los protones, por lo cual, el radio atómico del elemento también incrementa debido a su distribución espacial. Como ejemplo podemos tomar dos elementos del grupo I, el hidrogeno $\left({ }^{1} \mathrm{H}\right)$ y el cesio $\left({ }^{55} \mathrm{CS}\right)$. El primero es el elemento más simple, pertenece al período 1 y solamente tiene un electrón en un nivel electrónico; su radio atómico es de 1.10 ángstroms $(\AA)$, mientras que el segundo pertenece al período 6, tiene 55 electrones y seis niveles electrónicos; su radio atómico es de 3.43 A. Aún a pesar de este incremento en el radio atómico del elemento, es importante notar que si nos movemos en la tabla de izquierda a derecha (grupos 2, 3, 4, etcétera), el radio atómico comienza a disminuir; esto debido a que el incremento del número de protones en el núcleo también ejerce una mayor atracción electromagnética sobre los electrones en los orbitales. Como ejemplo podemos tomar dos elementos del período 6, el ${ }^{55} \mathrm{Cs}$ y el bismuto ${ }^{83} \mathrm{Bi}$ ). El primero, como se mencionó anteriormente, tiene 55 electrones distribuidos en sus seis orbitales y un radio atómico de $3.43 \AA$; mientras que el ${ }^{83} \mathrm{Bi}$, al pertenecer al grupo 15 (vA), tiene 83 electrones, es decir, 28 electrones y 28 protones más que el ${ }^{55} \mathrm{CS}$ y su radio atómico disminuye a un valor de $2.07 \AA$. Con esto podemos predecir que el radio atómico de los elementos aumenta al leer la tabla de arriba hacia abajo, y de derecha a izquierda, por lo que el Francio $\left({ }^{87} \mathrm{Fr}\right)$, del grupo 1, es el elemento con el mayor radio atómico, de $3.48 \AA$ (Aldersey-Williams, 2011).

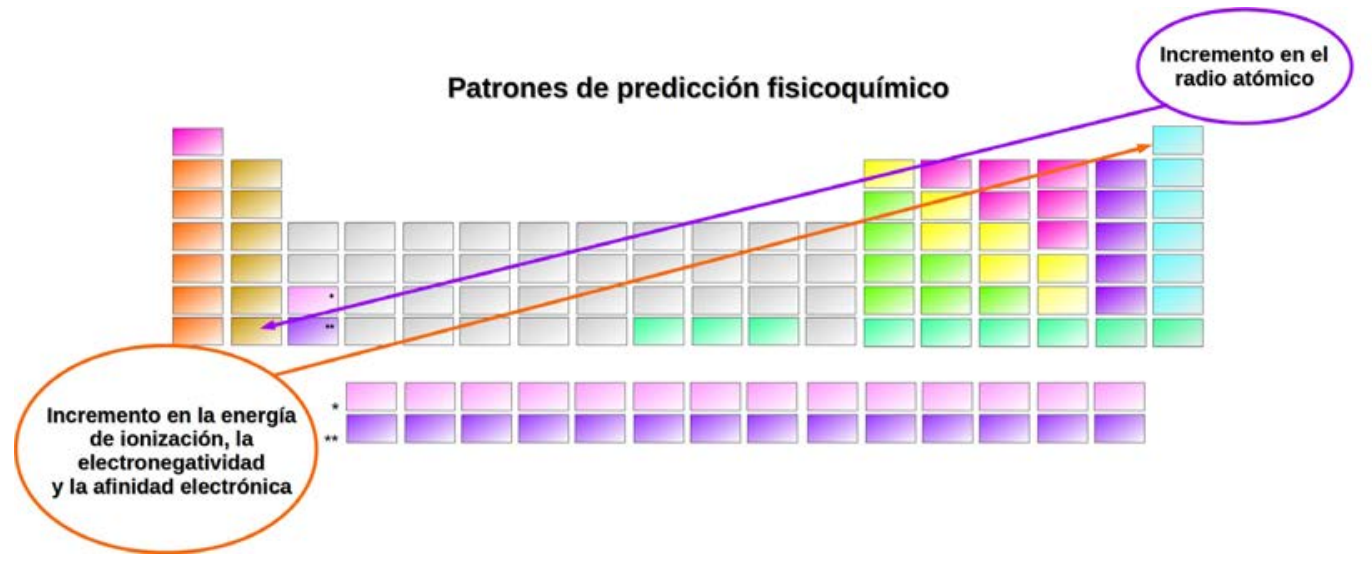

Otra propiedad predecible es el potencial de ionización, que se define como la cantidad de energía necesaria para arrancarle a un átomo, en su estado fundamental, el electrón más débilmente unido del último orbital. Asimismo, es importante señalar que la simetría en la configuración electrónica influye en la energía de ionización, por lo que los elementos del grupo 1 son a los que se les puede arrancar el electrón del orbital más alejado con la menor cantidad de energía. Para ejemplificar esta propiedad, podemos tomar dos elementos ubicados en los extremos de la tabla, el ${ }^{87} \mathrm{Fr}$ y el helio ( ${ }^{2} \mathrm{He}$ ). El primero, como se describió anteriormente, pertenece al grupo 1 y período 7, es decir, tiene un 
único electrón en el séptimo orbital, muy lejos del núcleo, por lo que, con muy poca energía de ionización (380 $\mathrm{kJ}_{\mathrm{Umol}}^{-1}$ ) es posible arrancarlo. Por otra parte, el ${ }^{2} \mathrm{He}$, que pertenece al grupo 18 (VIIA) y período 1, tiene un único orbital que está completo con dos electrones muy cerca del núcleo, por lo cual, su energía de ionización es la más alta de la tabla con un valor de $2372.3 \mathrm{kJUmol}^{-1}$. Con esto es posible predecir el patrón de comportamiento para la energía de ionización, el cual es contrario al del radio atómico, o sea, la energía de ionización aumentará en los elementos que se encuentren más a la derecha y más arriba de la tabla.

La afinidad electrónica y la electronegatividad son otras dos propiedades que nos proporcionan información del comportamiento fisicoquímico de los elementos. Éstas no tienen un comportamiento regular a lo largo de la tabla, sin embargo, aún es posible observar una tendencia de predicción al incrementar la energía de atracción electromagnética del núcleo sobre los electrones. Podemos definir la afinidad electrónica como la energía liberada cuando un átomo gaseoso en su estado fundamental captura un electrón para convertirse en un anión (un ión con carga negativa), mientras que la electronegatividad es la capacidad que tienen los átomos para atraer a los electrones cuando forman un enlace químico. La característica principal, para ambos casos, es el incremento en la energía electromagnética de atracción en los elementos con orbitales más cercanos al núcleo, o bien, dicho de otro modo, la afinidad electrónica y la electronegatividad serán mayores en los elementos con un menor radio atómico. Como ejemplo podemos considerar dos elementos ubicados en los extremos de la tabla, el ${ }^{87} \mathrm{Fr}$ y flúor $\left({ }^{9} \mathrm{~F}\right)$. El primero ya se tomó como ejemplo y sabemos que pertenece al grupo 1, período 7, y es el elemento con el mayor radio atómico (3.48 A), y una alta densidad electrónica. El ${ }^{9} \mathrm{~F}$ pertenece al grupo 17 (VIIA) y período 2, su radio atómico es menor (1.47 A) y contiene 9 electrones. El ${ }^{87} \mathrm{Fr}$ es un elemento con un radio atómico muy grande, además de que la alta densidad electrónica produce un apantallamiento y fuerzas de repulsión que evitan que otros electrones sientan la atracción del núcleo. Por otra parte, el ${ }^{9} \mathrm{~F}$ tiene un menor radio atómico, el núcleo ejerce una mayor atracción electromagnética sobre los electrones y debido a su configuración de valencia permite recibir un electrón más para completar su nivel y formar enlaces. Con esto es posible predecir el patrón de comportamiento para la afinidad electrónica y la electronegatividad, el cual aumentará de valor para los elementos que se encuentren más hacia la derecha y hacia arriba de la tabla periódica (Benfey y College, 2009).

\section{Conclusión}

La tabla periódica es una poderosa herramienta que organiza y clasifica los elementos de acuerdo con sus propiedades fisicoquímicas. Algunas de estas propiedades pueden predecirse por ciertos patrones, que están en función de la posición de los elementos. Una correcta interpretación de estos patrones ayudará, a los estudiantes y profesores, a comprender y relacionar la información contenida en la tabla, en vez de memorizarla. 


\section{Referencias}

* Aldersey-Williams, H. (2011). Periodic Tales: The Curious Lives of the Elements. Editorial Planeta, S. A.

* Benfey, T., College, G., y the Chemical Heritage Foundation. (2009). The Biography of a Periodic Spiral: From Chemistry Magazine, Via Industry, to a Foucault Pendulum. Bulletin for the History of Chemistry, 34(2), 141-145.

* Karol, P. J., Barber, R. C., Sherrill, B., Vardaci, E., Yamazaki, T. (2016). Discovery of the elements with atomic numbers $Z=113,115$ and 117 (IUPAC Technical Report). Pure and Applied Chemistry, 88(1-2), 139-153.

* Ramos M. (2017). Julius Lothar Meyer, EcuRed. https://www.ecured.cu/index. php?title=Julius_Lothar_Meyer\&oldid=3502780

- Scerri, E. R. (2007). The Periodic Table: Its Story and Its Significance. Journal of Chemical Education, 84(4), 598.

\section{Cómo CITAR ESTE ARTículo}

* Hernández Adame, Luis, Hernández-Adame, Pablo Luis, Ruiz-García, Jaime y VegaCarrillo,Héctor René.(2020, noviembre-diciembre). Latablaperiódicaysuspatrones para la predicción del comportamiento fisicoquímico. Revista Digital Universitaria (RDU), 21(6). Dol: http://doi.org/10.22201/cuaieed.16076079e.2020.21.6.5 